\title{
Precise Infrared Radial Velocimetry with the TripleSpec Exoplanet Discovery Instrument: Current Performance and Results
}

\author{
Philip S. Muirhead ${ }^{a}$, Jerry Edelstein ${ }^{b}$, Jason T. Wright ${ }^{c}$, David J. Erskine ${ }^{d}$, Matthew W. \\ Muterspaugh $^{e}$, Kevin R. Covey ${ }^{a}$, Mario R. Marckwordt ${ }^{b}$, Samuel Halverson $^{b}$, Daniel Mondo $^{b}$, \\ James P. Lloyd ${ }^{a}$ \\ ${ }^{a}$ Cornell University, Ithaca, NY, USA \\ ${ }^{b}$ University of California, Berkeley, CA, USA \\ ${ }^{c}$ The Pennsylvania State University, University Park, PA, USA \\ ${ }^{d}$ Lawrence Livermore National Laboratory, Livermore, CA, USA \\ ${ }^{e}$ Tennessee State University, Nashville, TN, USA
}

\begin{abstract}
The TripleSpec Exoplanet Discovery Instrument (TEDI) is optimized to detect extrasolar planets orbiting midto-late $\mathrm{M}$ dwarfs using the Doppler technique at infrared wavelengths. TEDI is the combination of a Michelson interferometer and a moderate-resolution near-infrared spectrograph, TripleSpec, mounted on the Cassegrain focus of the Palomar 200-inch Hale Telescope. Here we present results from observations of a radial velocity standard star and a laboratory source over the past year. Our results indicate that focus effects within the interferometer, combined with non-common-path errors between the ThAr calibration source and starlight, limit our performance to several $100 \mathrm{~m} / \mathrm{s}$. An upgraded version of TEDI, TEDI 2.0, will eliminate this behavior by mixing ThAr with starlight in a scrambled fiber before a redesigned interferometer with minimal focal effects.
\end{abstract}

Keywords: Extrasolar Planets, Radial Velocity, Infrared Astronomy

\section{MOTIVATION}

Efficiently attaining $1 \mathrm{~m} / \mathrm{s}$ of radial velocity (RV) precision with near-infrared (NIR) spectroscopy enables the following: (1) detection of potentially-habitable Earth-mass and larger exoplanets orbiting nearby mid-to-late M dwarf stars too faint for visible radial velocimetry, (2) expansion of the number of early $\mathrm{M}$ dwarfs targetable by visible RV surveys to those at greater distances increasing the yield of detected potentially-habitable planets, (3) verification and mass-measurement of exoplanet transit candidates observed to transit such M dwarfs, (4) detection of exoplanets around active stars and young stars with too much visible RV jitter, ${ }^{1}$ and with double-line NIR RV spectroscopy: (5) the accurate measurement of the individual masses of brown dwarf binaries and (6) the accurate measurement of the masses of young star binaries with too much visible RV jitter.

\section{THE TRIPLESPEC EXOPLANET DISCOVERY INSTRUMENT}

The TripleSpec Exoplanet Discovery Instrument (TEDI) is designed to efficiently measure radial velocities of $M$ dwarfs with $<10 \mathrm{~m} / \mathrm{s}$ of precision at infrared wavelengths. TEDI is the combination of a Michelson interferometer and a moderate-resolution spectrograph, TripleSpec $(R=\lambda / \Delta \lambda=2700)$, located at the Cassegrain focus of the 200 inch $(5 \mathrm{~m})$ Hale Telescope at Palomar Observatory in Southern California. ${ }^{2}$ The combination exploits the advantages a method known as externally dispersed interferometry (EDI) ${ }^{3}$ dispersed fixed-delay interferometer (DFDI) [4, e.g.], or dispersed-Fourier transform spectroscopy ${ }^{5}$ for NIR radial measurements. An optical path difference, or delay, added to one of the interferometer arms multiplies the stellar spectrum by a sinusoidal transmission comb, which both provides a radial velocity reference and heterodynes the narrow features of the

Further author information: (Send correspondence to P.S.M.)

P.S.M.: E-mail: muirhead@astro.cornell.edu

Ground-based and Airborne Instrumentation for Astronomy III, edited by lan S. McLean,

Suzanne K. Ramsay, Hideki Takami, Proc. of SPIE Vol. 7735, 77357X · @ 2010

SPIE $\cdot$ CCC code: $0277-786 X / 10 / \$ 18 \cdot$ doi: $10.1117 / 12.857196$

Proc. of SPIE Vol. 7735 77357X-1 
stellar spectrum such that they survive the moderate resolution of the spectrograph. The comb calibrates and heterodynes the entire wavelength coverage of TripleSpec, which includes the full $\mathrm{J}, \mathrm{H}$ and $\mathrm{K}$ bands (0.9 to 2.5 $\mu \mathrm{m})$ simultaneously by virtue of being only moderate resolution.

\section{THEORY}

A detailed explanation of externally dispersed interferometry is described elsewhere. ${ }^{3}$ What follows is a modified explanation specifically suited to the TEDI data product. Given wavenumber $\nu$, an intrinsic stellar spectrum $S_{\nu}$, a spectrograph response function $R_{\nu}$, and an interferometer delay of $\tau$, an individual TEDI spectrum, $I_{\nu, \tau}$, is described as:

$$
I_{\nu, \tau}=\left[S_{\nu}(1+\cos (2 \pi \tau \nu))\right] * R_{\nu}
$$

where $*$ indicates a convolution and $(1+\cos (2 \pi \tau \nu))$ represents the effect of the sinusoidal transmission comb introduced by the interferometer. To fully heterodyne the narrow absorption features of the intrinsic stellar spectrum, the delay $\tau$ must be slightly varied by $\Delta \tau$ to modulate the resultant spectra, analogous to shifting the phase of a local oscillator for heterodyned radio spectroscopy. This can be done by either tipping one of the interferometer mirrors such that different delays appear perpendicular to the dispersion direction of the spectrograph [4, e.g.], or by pistoning one of the mirrors with a piezo stack and taking spectra at each piston position. With TEDI, we use the latter, pistoning the mirror to keep the interferometer modulation within a pixel rather than across several pixels. This dramatically reduces the effects of flat-field and bias-subtraction errors on measuring the modulation.

If we rewrite $\tau$ as a 'bulk' delay plus a small shift $\left(\tau_{0}+\Delta \tau\right)$ and assume that $1 / \Delta \tau$ is small compared to a resolution element of the spectrograph, we can remove $\Delta \tau$ from the convolution integral and rewrite equation 1 as:

$$
I_{\nu, \tau_{0}, \Delta \tau}=A_{\nu}+B_{\nu} \cos (2 \pi \Delta \tau \nu)-C_{\nu} \sin (2 \pi \Delta \tau \nu)
$$

where,

$$
\begin{array}{r}
A_{\nu}=S_{\nu} * R_{\nu} \\
B_{\nu}=\left[S_{\nu} \cos \left(2 \pi \tau_{0} \nu\right)\right] * R_{\nu} \\
C_{\nu}=\left[S_{\nu} \sin \left(2 \pi \tau_{0} \nu\right)\right] * R_{\nu}
\end{array}
$$

$A_{\nu}$ is the spectrum of the star at the moderate-resolution of the spectrograph, referred to as the 'conventional' spectrum. $B_{\nu}$ and $C_{\nu}$ describe the heterodyned spectrum and contain the signal from the high-resolution stellar features. By stepping the delay of the interferometer, and taking spectra at each step, we fit $A_{\nu}, B_{\nu}$ and $C_{\nu}$ to the modulation at each pixel according to equation 2. We can then construct the complex visibility:

$$
B_{\nu}-i C_{\nu}=\left[S_{\nu} e^{-i 2 \pi \tau_{0} \nu}\right] * R_{\nu}
$$

Under a Doppler shift of the stellar spectrum $S_{\nu} \rightarrow S_{\nu+\Delta \nu}$, where $\Delta \nu$ is small compared to a resolution element of the spectrograph, the change in the complex visibility can be shown to be:

$$
B_{\nu}^{\prime}-i C_{\nu}^{\prime}=\left[B_{\nu}-i C_{\nu}\right] e^{-i 2 \pi \tau_{0} \Delta \nu}
$$

as a consequence of the shift theorem for Fourier transforms. Thus, a Doppler shift causes a change of the phase of the complex visibility. Since all wavelengths expect the same Doppler shift, the change in phase versus wavelength is expected to follow a simple curve $2 \pi \tau_{0} \Delta \nu=2 \pi \tau_{0} \Delta v \nu / c$, where $v$ is the radial velocity of the star. This curve cannot be replicated by simply shifting $A_{\nu}, B_{\nu}$, and $C_{\nu}$ together across the detector, since it 
is due to the relative shift between the interferometer comb and the stellar spectrum. This makes the measured radial velocity highly robust against changes in the slit illumination, shifts in the detector or a miscalibration of the wavelength solution of the detector. Errors in the wavelength solution can still introduce systematic RV errors, since $\nu$ enters equation 2, but the effect on Doppler precision is significantly less than for conventional high-resolution spectrosocpy. For example, If the delay difference between 2 epochs is $\Delta \tau=5 \mu \mathrm{m}$, then a miscalibration of the wavelength solution of one epoch's complex visibility by $2 \times 10^{-} 5 \mu \mathrm{m}$ will introduce $3 \mathrm{~m} / \mathrm{s}$ of Doppler error into the phase in equation 7. For TripleSpec, this amounts to roughly $1 / 5$ of a pixel. This is a dramatically lower tolerance than is required for conventional Doppler spectroscopy, which often requires wavelength calibration to less than $1 / 1000$ of a pixel for $3 \mathrm{~m} / \mathrm{s}$ of performance. ${ }^{6}$

The phase change is, however, nearly $100 \%$ degenerate with the delay of the interferometer. If $\Delta \tau$ in equation 2 is incorrectly measured, that error will manifest itself as a spurious Doppler shift. TEDI uses injected emission lines from a ThAr hollow cathode lamp overlapped with the starlight to accurately and precisely measure the delay, as well as provide a simultaneous wavelength solution to the detector. As the delay is stepped by $\Delta \tau$, the ThAr lines fluctuate in intensity on the spectrograph. With an accurate ThAr line list, ${ }^{7} \tau_{0}$ and $\Delta \tau$ are fully constrained by ThAr line fluctuations. However, if the ThAr lamp and the starlight 'see' different delays due to different illumination through the interferometer, this will introduce systematic radial velocity errors. For a typical $\tau_{0}$ of $1 \mathrm{~cm}$, a misestimation of $\Delta \tau$ by $0.1 \mathrm{~nm}$ will manifest itself as a $3 \mathrm{~m} / \mathrm{s}$ spurious Doppler shift. The bulk delay $\tau_{0}$ is chosen to maximize the modulation introduced by stepping the delay $\Delta \tau$, equivalent to maximizing the visibility: $\sqrt{B_{\nu}^{2}+C_{\nu}^{2}}$. This is achieved when the spacing of the interferometer comb peaks matches the width of the stellar lines. The width of stellar lines for main sequence stars is usually dominated by rotational broadening, however, the complex nature of M-type near-infrared spectra require that the delays be chosen based on modeling the visibility as a function of spectral type using synthetic spectra.

For Doppler shifts where $\Delta \nu$ is comparable to a resolution element of the spectrograph, such as those introduced by the barycentric motion of the Earth $(\sim 10 \mathrm{~km} / \mathrm{s})$ then the conventional spectrum $A_{\nu}$ must also be shifted. Fortunately, errors in the estimation of the conventional shift do not translate into systematic RV errors, as they would with conventional spectroscopy. An error in the conventional shift manifests itself as more scatter in the phase difference between two complex visibilities rather then as a systematic radial velocity error, because the phase change in $B_{\nu}-i C_{\nu}$ is being measured between the wrong pixels. When fitting the phase shift between Doppler velocities, the conventional shift is left as a free parameter shifted to reduce the scatter.

Since the radial velocity is proportional to a phase, there is a degeneracy arising from $2 \pi$ increments. The velocity associated with a $2 \pi$ phase change is very large $(\sim 10 \mathrm{~km} / \mathrm{s})$ compared to those produced by exoplanets, so this degeneracy can be ignored.

\section{OBSERVATIONS}

TEDI was commissioned in Dec of 2007 on the Hale telescope, and was subsequently awarded a total of 28 nights through the Cornell-Caltech Palomar agreement until Dec of 2009, with roughly half the time lost to bad weather. The nights were used primarily to characterize TEDI performance on bright $\mathrm{M}$ dwarfs known to have low radial velocity variation from optical measurements. In this paper, we discuss our results on the star GJ 411. GJ 411 is a nearby, bright $(\mathrm{K}=3.2) \mathrm{M} 2$ dwarf known to have less than $7.2 \mathrm{~m} / \mathrm{s}^{\mathrm{s}}$ of radial velocity jitter ${ }^{8}$ and less than $1 \mathrm{~km} / \mathrm{s}$ of stellar rotation, ${ }^{9}$ providing narrow lines for precise Doppler measurements.

Observations of GJ 411 showed systematic radial velocity errors which varied as a function of wavelength. To understand these effects, we built a fiber-fed 'star simulator' which recreates a telescope beam through TEDI with similar illumination across the entire TripleSpec bandpass. Illuminating the simulator with a $\mathrm{Kr}$ gas discharge lamp, we simulated repeated epochs on a star while varying parameters such as telescope pointing and focus. The data analysis, results and discussion follow.

\section{DATA ANALYSIS}

The TEDI data product consists of 10 TripleSpec spectra of the combined stellar and ThAr light, known as a 'phase set.' The interferometer delay is stepped by $\Delta \tau=0.25 \mu \mathrm{m}$ between each exposure. 10 steps of $0.25 \mu \mathrm{m}$ 

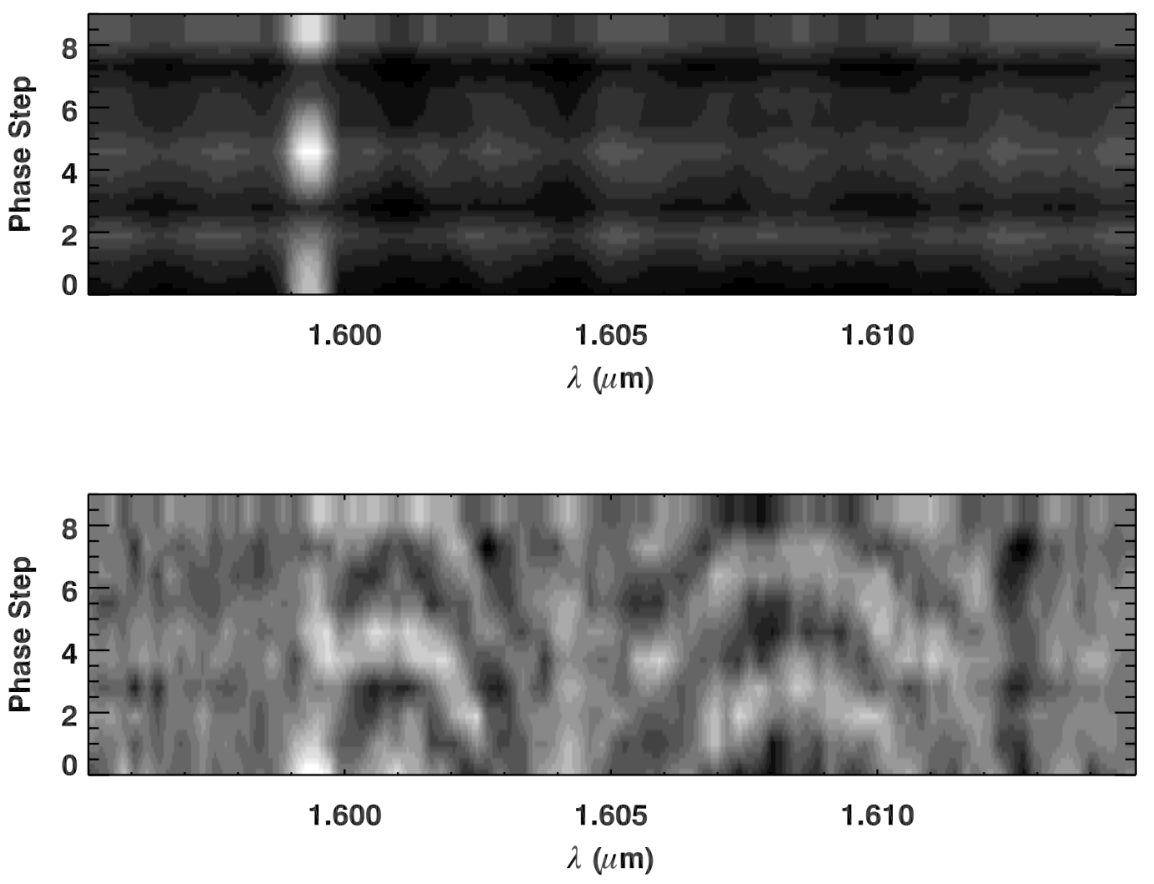

Figure 1. TEDI spectra of GJ 913 (M0 dwarf) vs wavelength and phase step for a narrow region of H band. The phase step value corresponds to a spectrum taken at that phase step of the interferometer, with all 10 spectra smoothed vertically for illustration. Top: The dominant signal is the ThAr emission line, and the stellar spectra underneath show fluctuations at each step due to changes in telescope pointing and seeing. Bottom: After fitting the ThAr line and correcting for stellar fluctuations, the modulation of the stellar light by the interferometer remains. $A_{\nu}, B_{\nu}$ and $C_{\nu}$ are fit to this pattern using equation 2. A small Doppler shift causes this pattern to shift dramatically versus phase step but only marginally versus wavelength. This makes Doppler shifts distinguishable from detector shifts, changes in the slit illumination or miscalibration of the detector wavelength solution.

provide full Nyquist sampling for fitting the coefficients $A_{\nu}, B_{\nu}$, and $C_{\nu}$ and constructing the complex visibility across the entire TripleSpec bandpass of 0.9 to $2.5 \mu \mathrm{m}$.

Each exposure consists of two interferometer outputs spread across the five TripleSpec orders. Before an observing night several dome flats and dome darks are taken. Highly variable and hot pixels are flagged using filters on the dome darks. The 3-sigma-clipped means of the flats and darks are used to create a single flat for each observing night. The interferometer outputs are piped to the spectrograph using fibers, and cannot efficiently be nodded along the slit, so an independent sky background is taken at each epoch. Since the Doppler signal is in the phase of the modulation introduced by the interferometer on each a pixel, an accurate sky/background subtraction is not critical to radial velocity measurements. After each exposure is background subtracted and flat-fielded, the exposures are converted to 1-dimensional spectra by interpolating the orders onto a rectilinear grid of wavelength vs. slit position using a stock TripleSpec wavelength solution. 1D spectra are extracted using either a sum across the slit dimension or by fitting a profile across the slit for each wavelength bin. ${ }^{10}$ The two methods show equivalent Doppler precision on the targets in this paper, since the targets, both stellar and laboratory sources, had high signal-to-noise, often in excess of 100.

The coefficients $A_{\nu}, B_{\nu}$ and $C_{\nu}$ are fit using a hybrid linear/non-linear Levenberg-Marquardt (LM) fitting sequence, similar to the rvlin algorithm for fitting planetary orbits to measured radial velocities. ${ }^{11}$ Since the phase steps $\Delta \tau$ will never be exactly $0.25 \mu \mathrm{m}$, each step is fit to the data using a LM algorithm. Within each non-linear LM iteration on the phase step sizes, a sinusoid is linearly fit to each pixel's modulation. The residuals from each LM iteration are the residuals from the sinusoidal fits to each pixel, which pressures the algorithm to find the phase step sizes that ensure sinusoidal modulation of the flux in each pixel. The sinusoidal modulations 
with phase-steps determine $A_{\nu}, B_{\nu}$ and $C_{\nu}$ according to equation 2 .

The most challenging aspect of the TEDI data analysis involves deconvolving the ThAr complex visibility from the stellar. Over the course of a phase set the stellar light fluctuates in intensity due to seeing and telescope pointing effects in addition to modulation by the interferometer, whereas the ThAr light is only modulated by the interferometer. Since the seeing image of the star is a slow function of wavelength, so are the non-interferometer intensity fluctuations, whereas the interferometer fluctuations vary with each resolution element.

To distinguish the ThAr from the starlight, a ThAr-only phase-set is taken near in time to the stellar phaseset, and a set of coefficients $A_{\nu}^{\mathrm{ThAr}}, B_{\nu}^{\mathrm{ThAr}}$ and $C_{\nu}^{\mathrm{ThAr}}$ are fit in the manner described. Fitting the coefficients to the star is similar, but involves two extra steps for each LM iteration on the phase step sizes: First, the expected ThAr fluxes are calculated at the phase step values for that iteration using the ThAr-only coeffiecients, and those fluxes are subtracted from the star plus ThAr fluxes. Second, changes in the stellar flux due to seeing and pointing are modeled using a median filter, which reveals slow changes in the stellar flux vs wavelength. The 10 stellar spectra are then normalized to correct for these variations before $A_{\nu}^{\text {star }}, B_{\nu}^{\text {star }}$ and $C_{\nu}^{\text {star }}$ are fit to the spectra using equation 2. Any errors in the phase-step sizes will leave behind residuals from the subtraction of the expected ThAr fluxes. When the star and residuals are normalized, the resultant spectra will not be sinusoidal versus phase step, providing pressure toward fitting the correct phase step sizes.

In the case of the Kr gas discharge lamp, the ThAr lines are well separated from the Kr lines, such that fitting the phase steps and $A_{\nu}^{\mathrm{Kr}}, B_{\nu}^{\mathrm{Kr}}$ and $C_{\nu}^{\mathrm{Kr}}$ is straightforward.

\section{GJ 411 Radial Velocity vs Wavelength}

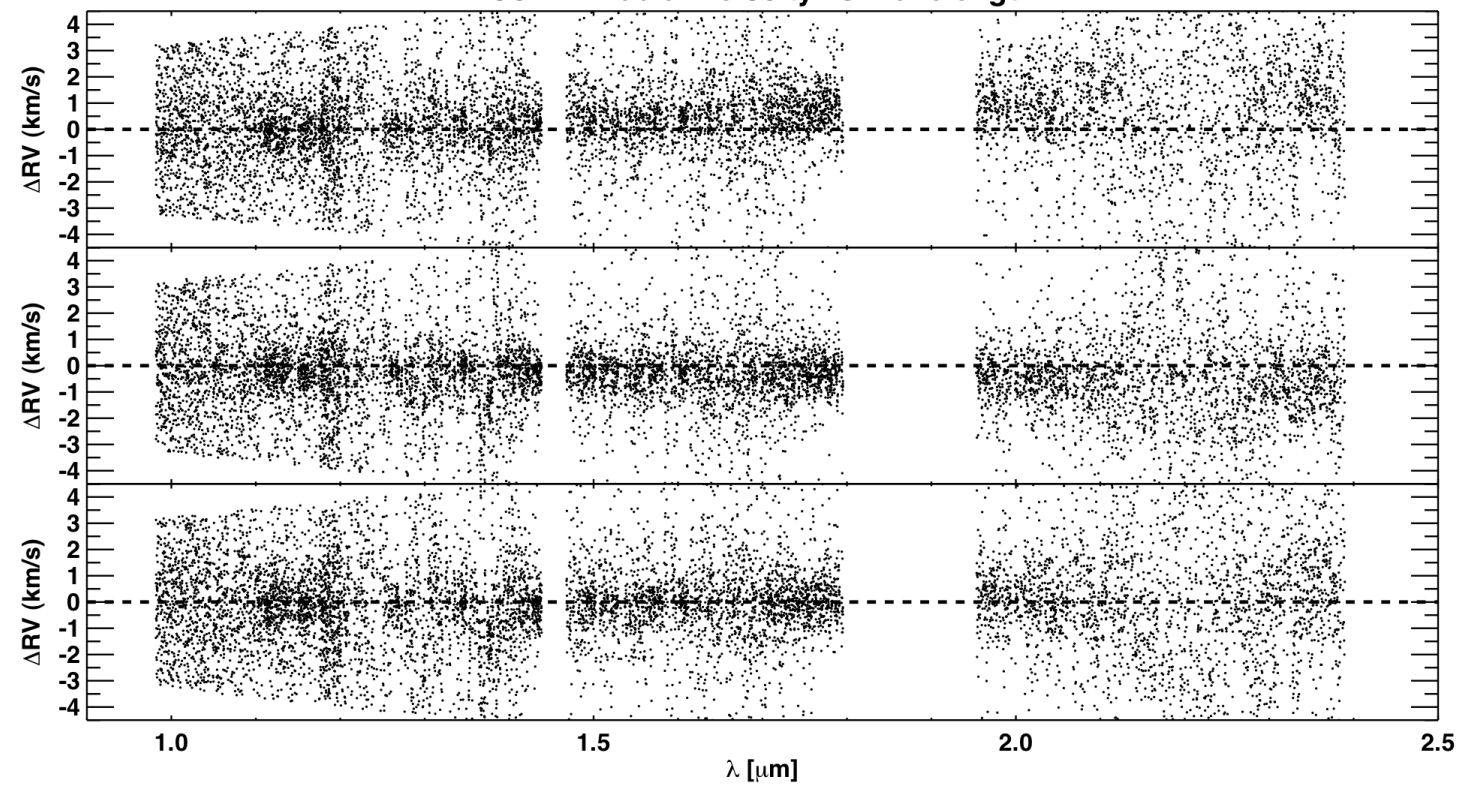

Figure 2. Radial Velocity versus wavelength as reported by each TEDI pixel for 3 epochs on GJ 411 taken back to back on the night of April 9th, 2009 (UT). Each epoch required roughly 6 minutes of observing time to complete. As expected, the values scatter about a $\Delta R V$ of $0 \mathrm{~km} / \mathrm{s}$, indicated on each plot with a dashed line. However there are clearly $\mathrm{km} / \mathrm{s}$ fluctuations above and below 0 , and the discrepancies are different for different wavelengths. The barycentric motion of the Earth relative to GJ 411 between these epochs was only $60 \mathrm{~m} / \mathrm{s}$, which does not explain the fluctuations. Experiments with a $\mathrm{Kr}$ source revealed the errors correlated with seeing and pointing changes through the interferometer.

\section{RESULTS}

Attempts to establish the intra-night and night-to-night radial velocity precision of TEDI on GJ 441 revealed systematic RV errors as a function of wavelength which fluctuated quickly with time. Figure 2 shows three plots 
of radial velocity versus wavelength for 3 epochs taken back-to-back during the night of April 9th, 2009 (UT). Attempts to understand and correct these fluctuations focused at first on the data analysis. It was not until we built a 'star simulator' telescope allowing for extensive off-telescope tests that we realized the errors were due to variations in the interferometer delays as seen by the star due to seeing and guiding errors.

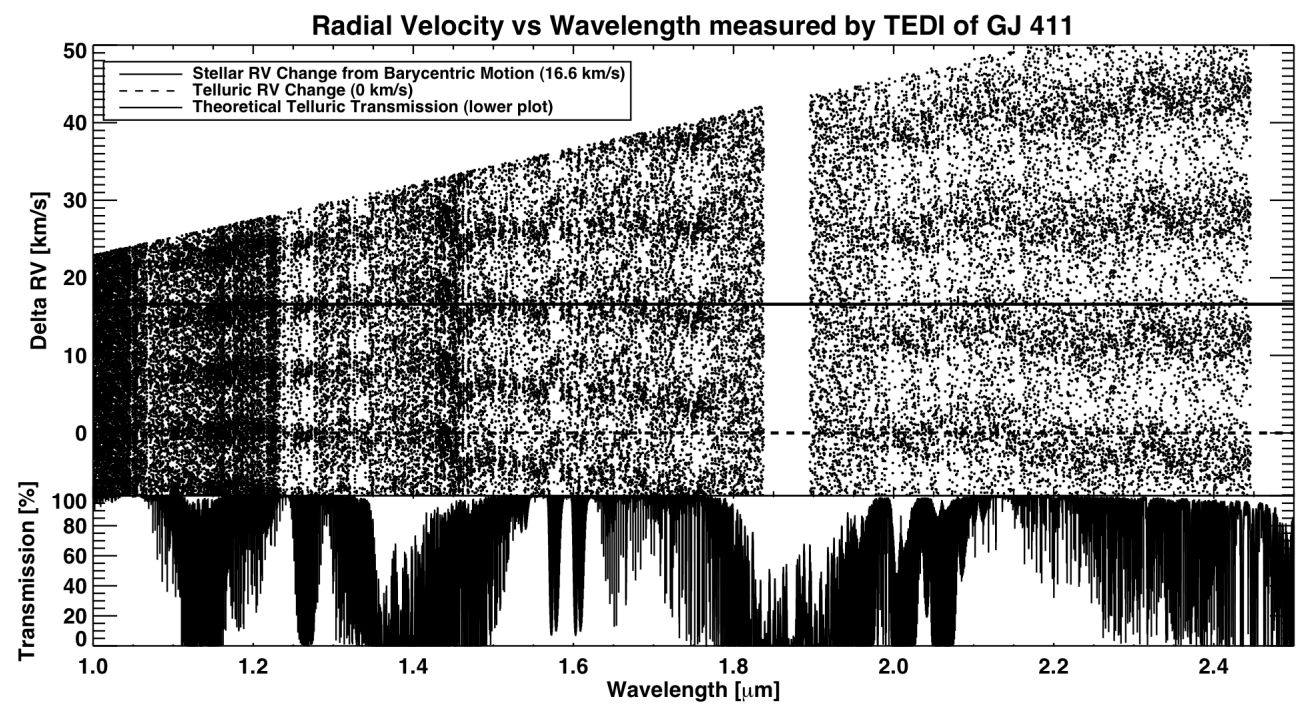

Figure 3. Plot showing the difference in measured radial velocity between two TEDI epochs of GJ 411 separated by about one month, along with a model of the telluric transmission lines introduced by the Earth's atmosphere. The barycentric motion of the Earth reliatve to GJ 411 changed by $16.6 \mathrm{~km} / \mathrm{s}$, and the regions dominated by stellar lines report that radial velocity change. Regions dominated by telluric lines report $0 \mathrm{~km} / \mathrm{s}$ as expected. The data is plotted several times to account for the $2 \pi$ degeneracy in the radial velocity. Systematic errors are still present and are clearest in $\mathrm{K}$ band, where the values show fluctuations about the correct value.

Nevertheless, an estimate of the radial velocity precision of TEDI was established. Figure 3 plots the change in radial velocity of GJ 411 between 2 runs separated by one month. The regions of the spectrum dominated by stellar lines report the correct barycentric velocity of the Earth relative to GJ 411 of $16.6 \mathrm{~km} / \mathrm{s}$. Regions dominated by the Earth's telluric lines report $0 \mathrm{~km} / \mathrm{s}$ as expected. Using the telluric dominated regions to calibrate the fluctuations, we estimate our night-to-night radial velocity stability to several $100 \mathrm{~m} / \mathrm{s}$ in $\mathrm{H}$ band.

Initial attempts to simulate starlight through TEDI involved a single $\mathrm{CaF}_{2}$ lens in a tube with a fiber feed. Simple lenses have inconsistent focal behavior across the bandwidth of TripleSpec, making it difficult to accurately simulate starlight from the telescope. To correct this, we constructed a star simulator telescope consisting of a 4-inch diamond-turned mirror with a fiber feed providing a consistent focus versus wavelength. This proved absolutely necessary to the discovery of TEDI's systematic errors, as they involve both dispersion and focal behavior.

Feeding the star simulator with a Kr gas discharge lamp, we discovered that slightly shifting the Kr beam in the interferometer plane introduced fluctuations identical to those seen in starlight. Shifting the Kr source by the equivalent of one arcsecond introduced $\mathrm{km} / \mathrm{s}$ of radial velocity error. The effect was also produced by sampling different parts of the image plane after the interferometer with TEDI's optical fibers. Figure 4 plots the change in measured radial velocity introduced by moving the image of the Kr fiber tip by the equivalent of 0.5 arcseconds on the sky.

\section{DISCUSSION}

Based on the experiments conducted with the Kr gas discharge lamp, the source of the radial velocity fluctuations in TEDI can be ascribed to two interrelated phenomena: (1) an image-plane dependence and wavelength- 


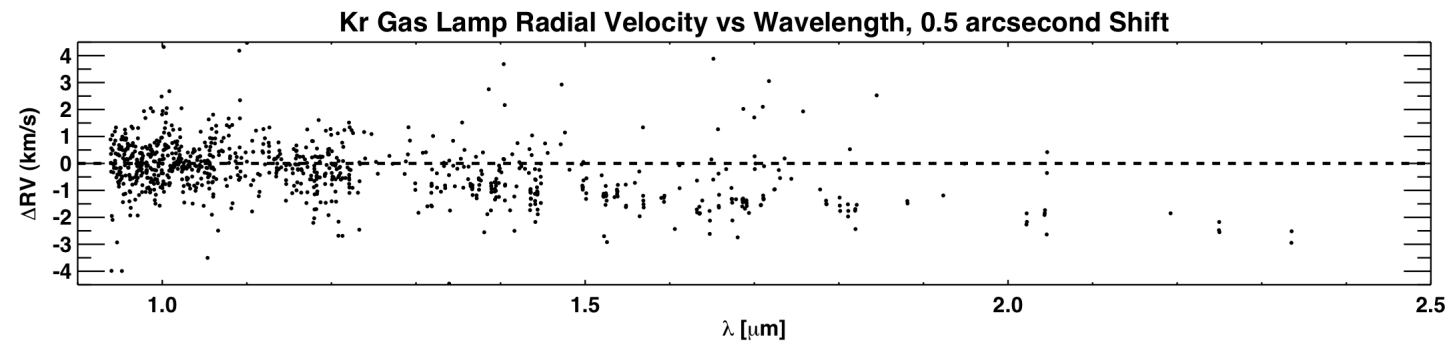

Figure 4. Plot showing the difference in measured radial velocity between two TEDI phase sets of a Krypton gas discharge lamp injected into TEDI with an illumination similar to starlight. Between phase sets the star simulator telescope was shifted to simulate a 0.5 arcsecond shift of starlight. The data points are rare longward of $1.5 \mu \mathrm{m}$ because the Kr lamp has relatively few emission lines in this region. There is a clear deviation from $0 \mathrm{~km} / \mathrm{s}$ (indicated in a dashed line) which was an effect isolated to translating the telescope.

dependence on the interferometer delay, which created (2) non-common-path errors between the delay calibration source (ThAr) and the starlight. The origin of the image-plane dependence is believed to come from the focal behavior through the interferometer. The starlight travels through the interferometer in a converging beam, coming to a focus on each of the cavity mirrors. If the starlight is not equally focused on the cavity mirrors, the interfering beams will have different radii of curvature when they recombine at the beam splitter. This leads to delay contours across the image when refocused onto the spectrograph slit. As the stellar image moves from seeing and pointing, the delay imprinted on the spectrum changes.

This effect would not be an issue if the delay calibration source, the ThAr emission lamp, shared the same illumination fluctuations as the starlight, experiencing the same wavelength-dependent delay fluctuations. Unfortunately, being an emission source, it does not share the same illumination fluctuations as the starlight, unlike absorption lines from a gas cell such as iodine which fully calibrate illumination changes. ${ }^{6}$

\section{TEDI 2.0}

To correct for the focal behavior and the non-common path errors, TEDI was disconnected from TripleSpec in December of 2009, and has been undergoing a substantial redesign. To eliminate non-common path errors between the starlight and the ThAr, the optical fibers have been relocated to mix starlight and ThAr light before injection into the interferometer. To eliminate the focal behavior of the interferometer, the optics have been redesigned to pass the mixed starlight and ThAr light through in a collimated beam. Great care has been taken to increase observing efficiency and overall throughput. A high-speed guider has been installed, utilizing a piezo tip/tilt mirror to pin the starlight to the tip of the input fiber with active throughput feedback.

TEDI 2.0 will be reattached to TripleSpec in June of 2010. A total of 15 nights in 2010 are dedicated to TEDI 2.0 performance development and radial velocity demonstration, before a dedicated survey for exoplanets around mid-to-late M dwarfs can begin in 2011.

\section{ACKNOWLEDGMENTS}

This work has been supported by the National Science Foundation under Grant No. AST-0505366, AST-096064 and by NASA Grant NNX09AB38G, as well as the NASA Earth and Space Sciences Fellowship Program.

\section{REFERENCES}

[1] Huélamo, N., Figueira, P., Bonfils, X., Santos, N. C., Pepe, F., Gillon, M., Azevedo, R., Barman, T., Fernández, M., di Folco, E., Guenther, E. W., Lovis, C., Melo, C. H. F., Queloz, D., and Udry, S., "TW ;Hydrae: evidence of stellar spots instead of a Hot Jupiter," A $\& A$ 489, L9-L13 (Oct. 2008). 
[2] Wilson, J. C., Henderson, C. P., Herter, T. L., Matthews, K., Skrutskie, M. F., Adams, J. D., Moon, D., Smith, R., Gautier, N., Ressler, M., Soifer, B. T., Lin, S., Howard, J., LaMarr, J., Stolberg, T. M., and Zink, J., "Mass producing an efficient NIR spectrograph," in [Society of Photo-Optical Instrumentation Engineers (SPIE) Conference Series], A. F. M. Moorwood \& M. Iye, ed., Society of Photo-Optical Instrumentation Engineers (SPIE) Conference Series 5492, 1295-1305 (Sept. 2004).

[3] Erskine, D. J., Edelstein, J., Feuerstein, W. M., and Welsh, B., "High-Resolution Broadband Spectroscopy Using an Externally Dispersed Interferometer," ApJL 592, L103-L106 (Aug. 2003).

[4] Zhao, B., Ge, J., and Groot, J., "The optical design of MARVELS spectrograph," in [Society of PhotoOptical Instrumentation Engineers (SPIE) Conference Series], Society of Photo-Optical Instrumentation Engineers (SPIE) Conference Series $\mathbf{7 4 4 0}$ (Aug. 2009).

[5] Behr, B. B., Hajian, A. R., Cenko, A. T., Murison, M., McMillan, R. S., Hindsley, R., and Meade, J., "Stellar Astrophysics with a Dispersed Fourier Transform Spectrograph. I. Instrument Description and Orbits of Single-lined Spectroscopic Binaries," ApJ 705, 543-553 (Nov. 2009).

[6] Butler, R. P., Marcy, G. W., Williams, E., McCarthy, C., Dosanjh, P., and Vogt, S. S., "Attaining Doppler Precision of 3 M s-1," PASP 108, 500-+ (June 1996).

[7] Kerber, F., Nave, G., and Sansonetti, C. J., "The Spectrum of Th-Ar Hollow Cathode Lamps in the 691$5804 \mathrm{~nm}$ region: Establishing Wavelength Standards for the Calibration of Infrared Spectrographs," ApJS 178, 374-381 (Oct. 2008).

[8] Endl, M., Cochran, W. D., Tull, R. G., and MacQueen, P. J., "A Dedicated M Dwarf Planet Search Using The Hobby-Eberly Telescope," AJ 126, 3099-3107 (Dec. 2003).

[9] Marcy, G. W. and Chen, G. H., "The rotation of M dwarfs," ApJ 390, 550-559 (May 1992).

[10] Horne, K., "An optimal extraction algorithm for CCD spectroscopy," PASP 98, 609-617 (June 1986).

[11] Wright, J. T. and Howard, A. W., "Efficient Fitting of Multiplanet Keplerian Models to Radial Velocity and Astrometry Data," ApJS 182, 205-215 (May 2009). 